\section{The Spt components of SAGA facilitate TBP binding to a promoter at a post-activator-binding step in vivo}

\author{
Aimée M. Dudley, Claire Rougeulle, ${ }^{1}$ \\ and Fred Winston ${ }^{2}$ \\ Department of Genetics, Harvard Medical School, \\ Boston, Massachusetts 02115 USA
}

The SAGA complex of Saccharomyces cerevisiae is required for the transcription of many RNA polymerase II-dependent genes. Previous studies have demonstrated that SAGA possesses histone acetyltransferase activity, catalyzed by the SAGA component Gen5. However, the transcription of many genes, although SAGA dependent, is Gen 5 independent, suggesting the existence of distinct SAGA activities. We have studied the in vivo role of two other SAGA components, Spt3 and Spt20, at the wellcharacterized GAL1 promoter. Our results demonstrate that both $\mathrm{Spt} 3$ and $\mathrm{Spt} 20$ are required for the binding of TATA-binding protein but not of the activator Gal4 and that this role is Gen5 independent. These results suggest a coactivator role for $S p t 3$ and $S p t 20$ in the recruitment of TBP.

Received September 8, 1999; revised version accepted September 28, 1999.

In eukaryotes, transcription initiation by RNA polymerase II (Pol II) requires not only the activities of the general transcription factors and gene-specific activators but also the activities of large coactivator complexes (Hampsey 1998; Hampsey and Reinberg 1999). The SAGA (Spt/ Ada/ㅁcn5/acetyltransferase) complex of Saccharomyces cerevisiae is one such coactivator complex. It is required for the full transcriptional activity of a subset of Pol II-dependent genes (Hampsey 1997; Grant et al. 1998). SAGA contains $>20$ proteins, including Gcn5, a histone acetyltransferase (HAT) whose activity has been shown to be required for the transcription of a subset of genes in vivo (Kuo et al. 1998; Wang et al. 1998). Recent studies have implicated Gcn5 in the recruitment of activators to promoters by SAGA (Cosma et al. 1999).

In addition to the HAT activity of Gen5, genetic and biochemical studies suggest that SAGA possesses other activities important for transcription (Horiuchi et al. 1997; Roberts and Winston 1997; Dudley et al. 1999; Sterner et al. 1999). In this paper we focus on two other

[Key Words: SAGA complex; S. cerevisiae; transcription; Spt3; TBP] ${ }^{1}$ Present address: Unité de Génétique Moléculaire Murine, Unité de Recherche Associeé-Centre National de la Recherche Scientifique (URA-CNRS) 1968, Institute Pasteur, 75724 Paris Cedex 15, France. ${ }^{2}$ Corresponding author.

E-MAIL Winston@rascal.med.Harvard.edu; FAX (617) 432-3993. components of SAGA, Spt20, and Spt3. Spt20 is required for the integrity of SAGA, as the complex cannot be detected in spt20s mutants (Grant et al. 1997). Spt3 is probably required for a subset of SAGA activities that are independent of Gcn5 and histone acetylation because SAGA purified from an spt3s mutant still possesses Gcn5-dependent HAT activity, yet spt3s mutants have multiple transcriptional defects (Roberts and Winston 1997; Dudley et al. 1999; Sterner et al. 1999|. Previous genetic and biochemical studies have shown that Spt3 interacts with TATA-binding protein (TBP) (Eisenmann et al. 1992; Lee and Young 1998) and suggest that Spt3 is structurally similar to particular TBP-associated factors (TAFs) (Birck et al. 1998). These findings suggest a model in which Spt3 plays a role in the binding of TBP to particular promoters in vivo.

To better understand the mechanism by which the Spt components of SAGA facilitate the transcription of a subset of RNA Pol II-dependent genes, we chose to study the effects of SAGA mutations on activation by Gal4 at the well-characterized GAL1 promoter. Studies of activation of the GAL promoters by Gal4 have served as a model system for transcriptional regulation for many years (Johnston and Carlson 1992; Ptashne and Gann 1997; Zaman et al. 1998). Furthermore, the GAL1 promoter is useful for such studies because its regulation is well characterized and it has a relatively simple promoter structure. Under inducing conditions, two proteins, TBP and Gal4, are known to bind the GAL1 promoter (Johnston and Carlson 1992). Thus, of the genes known to be regulated by the Spt components of SAGA (Roberts and Winston 1997; Sterner et al. 1999), the GAL promoters are the only ones for which the Spt dependence of both TBP binding and activator binding can be measured.

In this study we use chromatin immunoprecipitation assays to determine which steps in transcriptional activation require Spt20 and Spt3 at the GAL1 promoter. Our results demonstrate a severe reduction in TBP binding at the GAL1 TATA in spt3 $\Delta$ and spt20 2 mutants but only a modest decrease in a gcn $5 \Delta$ mutant. These results correlate well with the effects on GAL1 transcription in these mutants. In contrast, the transcriptional activator Gal4 is bound to the GAL1 upstream activating sequence $\left(\mathrm{UAS}_{\mathrm{G}}\right)$ in all three SAGA mutants. Furthermore, the acetylation of histone $\mathrm{H} 3$ at the GAL1 promoter is only slightly decreased in $g c n 5 \Delta$, spt3s, and spt20s mutants. Taken together, our results suggest a Gcn5-independent activity for the Spt20 and Spt 3 components of SAGA that is required for the recruitment of TBP, but not of activators, to a subset of Pol II-dependent promoters in vivo.

Results

GAL1 transcription is dependent on Spt3 and Spt20, but not Gcn5

To understand the role of different SAGA functions, we 
examined the effects of mutations in three different classes of SAGA genes, SPT3, GCN5, and SPT20, on the well-studied GAL1 gene of $S$. cerevisiae. Spt3 and Gcn5 are probably required for distinct aspects of SAGA function, whereas Spt20 is believed to be required for all SAGA function (Grant et al. 1997; Sterner et al. 1999). The GAL1 promoter was particularly useful for analysis of Spt3 and Spt20 because gcn5s mutants are $\mathrm{Gal}^{+}$, whereas spt $3 \Delta$ are moderately $\mathrm{Gal}^{-}$, and spt20 mutants are tight $\mathrm{Gal}^{-}$(Roberts and Winston 1997; Sterner et al. 1999). To study primary events of transcriptional activation at the GAL1 promoter, we analyzed cells at an early point in galactose induction, sufficient for the wild-type strain to have fully induced expression (Materials and Methods). At this point in galactose induction, GAL1

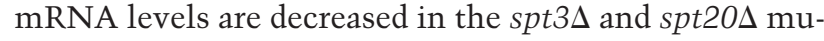
tants by $\sim 50$-fold relative to wild-type levels (Fig. 1). In contrast, a gcn $5 \Delta$ mutant shows only a modest 2.5 -fold decrease in GAL1 mRNA levels. Thus, induction of the GAL1 promoter is strongly dependent on the Spt3 and Spt20 components of SAGA and only mildly dependent on the Gen5 HAT.

\section{TBP binding at GAL1 is defective in $\mathrm{spt} 3 \Delta$}

\section{and spt20د mutants}

An important step in transcription initiation is the recruitment of TBP to the TATA region of a promoter. Previous studies of the GAL1 promoter in vivo demonstrated that TBP is absent from the GAL1 TATA region under conditions of both glucose repression or noninduction (raffinose or glycerol). However, upon induction with galactose, TBP associates strongly with the GAL1 TATA region (Selleck and Majors 1987a,b; Kuras and Struhl 1999; Li et al. 1999). To test whether SAGA mutations affect the binding of TBP to the GAL1 TATA in vivo, we used the chromatin immunoprecipitation assay (Dedon et al. 1991; Orlando and Paro 1993; Strahl-Bolsinger et al. 1997). We observed a strong (>10-fold) defect in the ability of TBP to bind the GAL1 TATA region in

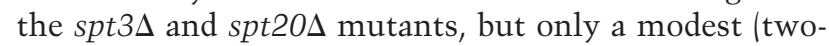

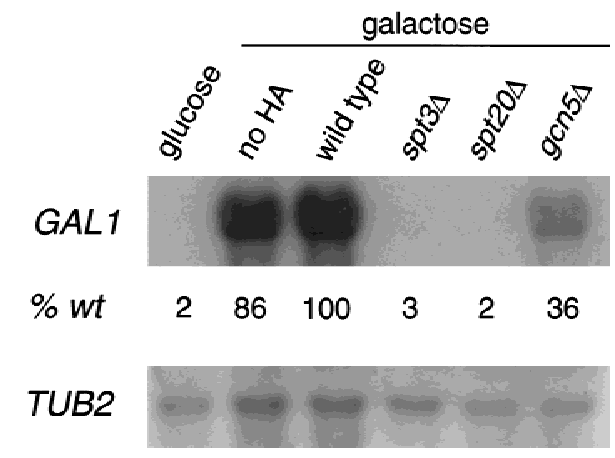

Figure 1. GAL1 mRNA levels in three classes of SAGA mutants. Northern hybridization analysis was performed on total yeast RNA prepared from glucose-repressed wild-type strains and galactose-induced wild-type, spt3s, spt20s, and gcn5s strains. Quantitation was performed by PhosphorImager analysis (Molecular Dynamics). fold) decrease in TBP binding in the gcn5 5 mutant (Fig. 2). These results strongly suggest that the spt3s and spt20D defects in GAL1 activation are caused by an inability to stably bind TBP to the promoter.

\section{Gal4 binding is unaffected in spt3s and spt $20 \Delta$ mutants}

One condition that could account for both the defect in GAL1 transcription and in TBP binding is the inability of the transcriptional activator Gal4 to bind to $\mathrm{UAS}_{\mathrm{G}}$ in the spt mutants. To test this hypothesis, we used chromatin
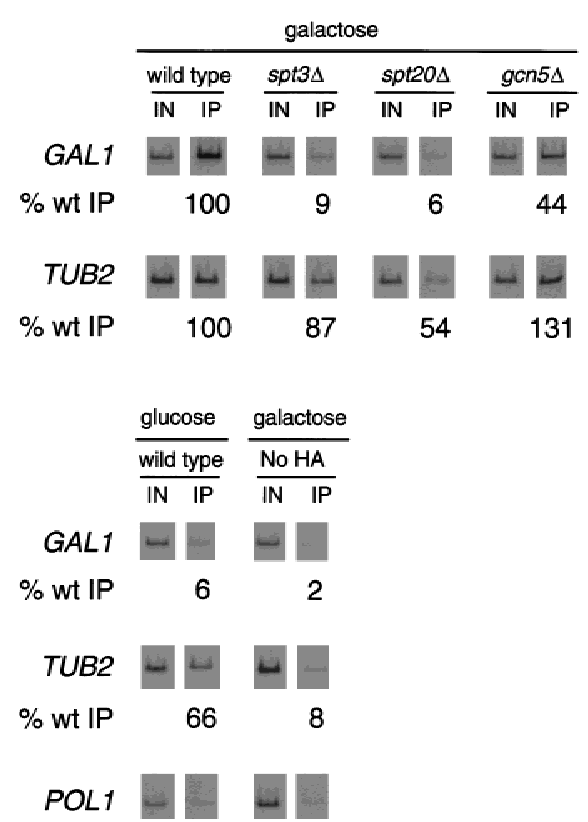

Figure 2. TBP is not bound to the GAL1 TATA in spt3A and spt20A mutants. Chromatin immunoprecipitation was performed in parallel with RNA analysis (Fig. 1) from glucose-repressed wild-type strains and galactose-induced wild-type,

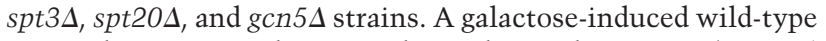
strain that contained TBP without the triple HA tag (no HA) was included as a negative control for the immunoprecipitation. TBP was immunoprecipitated using the 12CA5 antibody against the HA epitope. PCR products correspond to the GAL1 TATA region (GAL1), the TUB2 TATA region (TUB2), or the $P O L 1$ open reading frame as a negative control. The percentage of DNA immunoprecipitated (\% wt IP) in each of the mutants was normalized to the amount immunoprecipitated from the galactose-induced wild-type strain. One set of PCR reactions is shown, and the quantitation represents the average of several experiments. The average values with standard errors for the measurements of TBP binding to the GAL1 TATA on galactose-

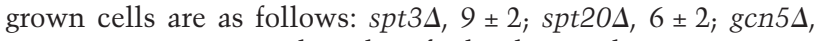
$44 \pm 3$; No HA, $2 \pm 1$. The values for binding to the TUB2 TATA on galactose-grown cells are: $s p t 3 \Delta, 87 \pm 34$; spt $20 \Delta, 54 \pm 10$; $g c n 5 \Delta, 131 \pm 22$; No HA, $8 \pm 2$. The values for wild-type glucose-grown cells are as follows: GAL1, $6 \pm 3$; TUB2, $66 \pm 8$. The low level of DNA detected in the No-HA and the POL1 PCR reactions represents the low amount of TBP-independent DNA precipitated as background in this assay. The POL1 negative control was performed on all samples, and the results were essentially the same as the example shown. 
immunoprecipitation assays to analyze the occupancy of the $\mathrm{UAS}_{\mathrm{G}}$ by Gal4 (Fig. 3). Interestingly, in all the SAGA mutants tested we observed in vivo occupancy of the UAS $_{\mathrm{G}}$ by Gal4 at levels similar to that of wild type. Therefore, Spt3 and Spt20 are not required for Gal4 binding. Together with the defects in TBP binding in spt3A and spt20s mutants, these results strongly suggest that Gal4 cannot recruit TBP in the absence of Spt3 or Spt20.

Histone $H 3$ acetylation in $\operatorname{gcn} 5 \Delta, \operatorname{spt} 3 \Delta$,

and spt20s mutants

Previous studies have suggested that Spt3 functions in SAGA independently of the HAT activity of Gcn5 (Roberts and Winston 1997; Dudley et al. 1999; Sterner et al. 1999|. To test directly whether spt3s, gcn5s, or spt20s

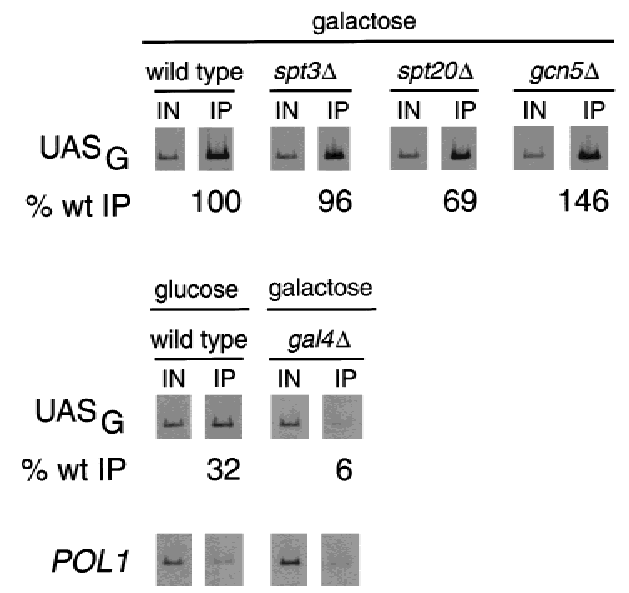

Figure 3. Gal4 occupies the $\mathrm{UAS}_{\mathrm{G}}$ in SAGA mutants. Chromatin immunoprecipitation was performed in parallel with RNA analysis (Fig. 1) from glucose-repressed wild-type strains and galactose-induced wild-type, spt3s, spt20s, or gcn5s strains. A galactose-induced gal4s strain was included as a negative control for the immunoprecipitation. Gal4 was immunoprecipitated using the RK5Cl antibody against Gal4 (Santa Cruz Biotechnology). PCR products correspond to the $\mathrm{UAS}_{\mathrm{G}}$ or the POL1 ORF as a negative control. The percentage of $\mathrm{UAS}_{\mathrm{G}}$ DNA immunoprecipitated (\% wt IP) from each of the mutants was normalized to the amount precipitated from the galactoseinduced wild-type strain. One set of PCR reactions is shown, and the quantitation represents the average of several experiments. The average values with standard errors for the measurements of Gal4 binding to the $\mathrm{UAS}_{G A L}$ on galactose-grown cells are as follows: spt $3 \Delta, 96 \pm 6$; spt20s, $69 \pm 1$; gcn5 $\Delta, 146 \pm 12$; gal4s, $6 \pm 1$. The measurement for wild type grown on glucose was $32 \pm 4$. The low level of DNA detected in the gal4s and the POL1 PCR reactions represents the low amount of Gal4-independent DNA precipitated as background in this assay. The POL1 negative control was performed on all samples, and the results for all the other extracts were essentially the same as the example shown. In this experiment we detected a modest, but significant, threefold increase in Gal4 binding to the $\mathrm{UAS}_{\mathrm{G}}$ in galactose-induced cells relative to glucose-repressed cells. These results differ slightly from previous in vivo footprint analyses that detected little or no Gal4 occupancy of the UAS in glucose-repression conditions (Giniger et al. 1985; Selleck and Majors 1987b), a result that highlights the sensitivity of the chromatin immunoprecipitation assay. mutations alter histone acetylation at the GAL1 promoter, we used chromatin immunoprecipitation assays to determine the histone $\mathrm{H} 3$ acetylation levels of GAL1 in these SAGA mutants (Fig. 4). As a control, we examined the $\mathrm{H} 3$ acetylation levels at HIS3 and observed a three-fold decrease in a gcn $5 \Delta$ mutant, consistent with previously published results (Kuo et al. 1998). At GAL1, our results show that $\mathrm{H} 3$ acetylation is only mildly re-

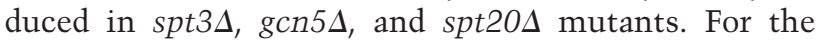
gcn $5 \Delta$ mutant, this effect correlates with the weak effect on GAL1 transcription. However, for the spt3s and spt20s mutants, there is not a good correlation between $\mathrm{H} 3$ acetylation and the severe GAL1 transcriptional defects. This result strongly suggests that GAL1 transcription is not significantly dependent on histone acetylation by SAGA and that the role of Spt3 is unrelated to Gcn5-dependent histone acetylation.

\section{Discussion}

Coactivators are believed to act as intermediaries between gene-specific activators and TBP or other general transcription factors. However, the molecular mechanisms by which coactivators function are not well understood (Hampsey 1998; Hampsey and Reinberg 1999). Our experiments have defined an in vivo coactivator function for Spt3 and Spt20 of the SAGA complex. In both spt3s and spt20s mutants, Gal4 binding is normal, but TBP fails to bind to the GAL1 promoter, and transcription is reduced $>50$-fold. Thus, the Spt 3 and Spt 20

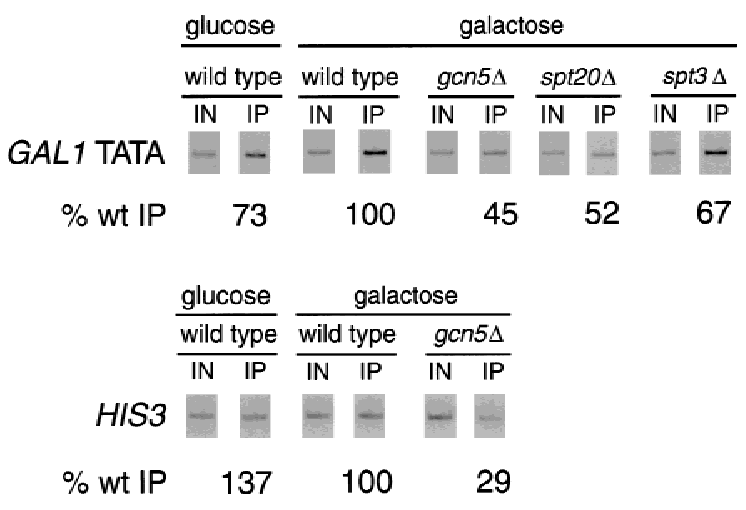

Figure 4. Histone $\mathrm{H} 3$ acetylation in $\operatorname{spt} 3 \Delta, g c n 5 \Delta$, and spt20s mutants. Chromatin immunoprecipitation was performed in parallel with RNA analysis (Fig. 1) from glucose-repressed, wildtype strains and galactose-induced wild-type, spt3s, spt20s, or gcn5 $\Delta$ strains. Histone H3 acetylated at Lys-9 and Lys-14 was immunoprecipitated using antisera previously described (Kuo et al. 1998). PCR products correspond to the GAL1 TATA or the HIS3 promoter as a control. The percentage of GAL1 TATA DNA immunoprecipitated (\% wt IP) from each of the mutants was normalized to the amount precipitated from the galactoseinduced wild-type strain. One set of PCR reactions is shown, and the quantitation represents the average of several experiments. The spt $3 \Delta$ and spt $20 \Delta$ mutants showed some variation, $40 \%-110 \%$ for spt $3 \Delta$ and $36 \%-70 \%$ for spt $20 \Delta$. At HIS3, a threefold decrease in $\mathrm{H} 3$ acetylation level was observed in the gcn5 $\Delta$ mutant compared with wild type, similar to previously reported results (Kuo et al. 1998). 
proteins are required for Gal4 to recruit TBP to the GAL1 promoter. These results constitute the first in vivo demonstration of such a coactivator role. Our results fit well with other studies of Gal4 and TBP binding in vivo that demonstrated that TBP binding requires the binding and activation of Gal4 (Selleck and Majors 1987a,b; Kuras and Struhl 1999; Li et al. 1999).

The model most consistent with these results is one in which TBP is physically recruited to the GAL1 promoter by the Spt components of SAGA. Previous analysis suggests a physical interaction between Spt3 and TBP (Eisenmann et al. 1992; Lee and Young 1998). In addition, the positions of amino acid changes of both TBP and Spt3 mutants that alter their functional interaction suggest specific regions of each protein that may be involved in TBP-Spt3 interactions (Eisenmann et al. 1992). These results are supported by recent studies of TBP-TAF 28 interactions (Lavigne et al. 1999), as Spt3 is predicted to have structural similarity to both $\mathrm{TAF}_{\mathrm{II}} 18$ and $\mathrm{TAF}_{\mathrm{II}} 28$ (Birck et al. 1998). Recent results have shown that SAGA can be recruited by several transcriptional activators including VP16 (Ikeda et al. 1999), Gcn4 (Natarajan et al. 1998), and Rtg3 (Massari et al. 1999). Taken together with these results, our data suggest a model for coactivation in which SAGA is targeted to the GAL1 promoter by interactions with Gal4, followed by recruitment of TBP via interactions with Spt3. The recruitment of TBP by the Spt proteins may be followed by the establishment of previously demonstrated interactions between Gal4 with certain general transcription factors, including TBP, TFIIB, and Srb4 (Melcher and Johnston 1995; Wu et al. 1996; Koh et al. 1998).

Our results, together with previous studies, suggest that other SAGA components may be required for this coactivator function. Spt20, Spt7, and Ada1 are all probably required for the integrity of SAGA (Grant et al. 1997; Sterner et al. 1999). Therefore, the defect observed in spt20 $\Delta$ mutants is probably caused by the loss of all SAGA functions, including Spt3, although we cannot rule out a more direct role. In addition to Spt3, the Spt8 protein has been implicated in SAGA-TBP interactions, and in vitro data suggest a direct role for Spt8 (Sterner et al. 1999). These results suggest that Spt8, along with Spt3, might be required for TBP binding at the GAL1 promoter. However, an spt $8 \Delta$ mutant is $\mathrm{Gal}^{+}$, suggesting that Spt8 plays, at most, a minor role at this promoter. Furthermore, previous studies have demonstrated that a particular mutation in SPT3 can partially bypass the requirement for Spt8, suggesting that Spt8 plays a more auxiliary role (Eisenmann et al. 1994). Possibly, both Spt3 and Spt8 assist TBP recruitment, but they contribute to different degrees in a promoter-specific fashion. In addition to these Spt proteins, other SAGA components, including the Ada and Taf proteins, may play related roles in the assembly of the preinitiation complex. Finally, recent evidence suggests that the Snf/Swi complex also helps to activate transcription at a step subsequent to activator binding (Ryan et al. 1998).

Our results cannot rule out a model in which the Spt components of SAGA act at another step in transcrip- tional activation that results in the recruitment of TBP. For example, the Spt proteins could facilitate some aspect of Gal4 activation, subsequent to its DNA binding, that allows Gal4 to recruit TBP to the GAL1 promoter. Such a role could include helping to determine the correct interactions of Gal4 with either Gal80 or Gal3, both known to interact with Gal4 under inducing conditions (Blank et al. 1997; Yano and Fukasawa 1997). Given that only particular promoters are dependent on the Spt proteins, it is likely that multiple factors determine both their requirement and allowing their function at any Sptdependent promoter.

Finally, our results demonstrate that the function of SAGA at GAL1 is largely independent of the Gen5 HAT. These results provide an interesting contrast to those from a recent study that demonstrated a strong Gcn5 requirement for the binding of the Swi4 activator to the HO promoter in vivo (Cosma et al. 1999). The differences between these two sets of results demonstrate that different promoters can require distinct SAGA components for mechanistically distinct functions. The determinants of these requirements at any promoter are an issue for future investigation.

\section{Materials and methods}

S. cerevisiae strains

All S. cerevisiae strains are isogenic to a $G A L 2^{+}$derivative of S288C (Winston et al. 1995). Strains were constructed with the following relevant genotypes: wild type (FY1887 and FY1888), spt3-202 (FY1889 and

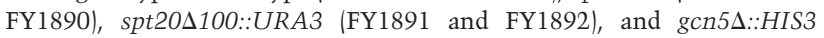
(FY1893 and FY1894). Each of these strains also contained an spt15 $::$ LEU2 mutation in the genome and an $H_{3}-S P T 15$ TRP1 CEN plasmid (Kuras and Struhl 1999) as the only source of wild-type TBP. The $H A_{3}-S P T 15$ construct fully complemented the spt15 102::LEU2 mutation for all phenotypes tested, including growth on galactose (data not shown). The control strain for the Gal4 chromatin immunoprecipitation experiments, FY760 (ga14A::LEU2), was SPT15 ${ }^{+}$in the genome and contained no plasmid. The control strain for the TBP chromatin immunoprecipitation experiments, FY1886 (the "no-HA" control), contained spt15 $102:: L E U 2$ in the genome and contained $S P T 15^{+}$on a plasmid (Kuras and Struhl 1999) as the only source of wild-type TBP. Glucoserepressed strains were grown in YPD ( $2 \%$ glucose). Galactose-induced strains were grown in YPRaf ( $2 \%$ raffinose) and induced for $20 \mathrm{~min}$ by the addition of galactose to $2 \%$. RNA for Northern analysis and chromatin extracts were prepared from the same cultures grown to cell densities of $1 \times 10^{7}-2 \times 10^{7}$ cells $/ \mathrm{ml}$. Northern analysis and chromatin immunoprecipitation assays were performed on both sets of isogenic strains.

Northern hybridization analysis

Total yeast RNA was prepared as described previously (Swanson et al 1991). Northern blot analysis was performed on both sets of strains used for chromatin immunoprecipitations. One of the experiments is shown (Fig. 1), and the quantitation represents the average of both sets of strains. The GAL1 (St. John and Davis 1981) and TUB2 (Som et al. 1988) probes have been described previously.

\section{Chromatin immunoprecipitation}

Formaldehyde cross-linking extracts were prepared essentially as described previously (Kuras and Struhl 1999) with the following exceptions: First, all centrifugations to pellet the chromatin extract were performed for $1 \mathrm{~min}$ at 14,000 rpm in an Eppendorf centrifuge. Second, the separation of soluble chromatin following sonication was accomplished by a 1-hr centrifugation at $14,000 \mathrm{rpm}$ in an Eppendorf centrifuge. Immunoprecipitations of $\mathrm{HA}_{3}$-tagged TBP were performed as described previously (Kuras and Struhl 1999). Gal4 immunoprecipitations were performed by the same method used for the $\mathrm{HA}_{3}$-tagged TBP with the exception that binding was done in FA lysis buffer containing $150 \mathrm{~mm} \mathrm{NaCl}$ (Kuras and 
Struhl 1999) and washes were done three times in the same buffer and once in TE (10 mм Tris-HCl, 1 mм EDTA at pH 8.0). Immunoprecipitation of the hyperacetylated form of histone $\mathrm{H} 3$ was performed as described previously (Kuo et al. 1998). PCR reactions were performed essentially as described previously (Kuras and Struhl 1999), with the exception that PCR products were detected by the incorporation of $\left[{ }^{33} \mathrm{P}\right] \mathrm{dATP}$ in the reaction. The PCR primers amplify the following regions whose coordinates are given relative to the ATG (+1): GAL1 TATA primers amplify a 244-bp region from -190 to +54 ; GAL1 UAS primers amplify a 260-bp region from -536 to -276 ; TUB2 TATA primers amplify a 273-bp region from -186 to +87 ; POL1 ORF primers amplify a 219-bp region from +2499 to +2717 ; and HIS3 primers amplify a 105 -bp region from -28 to +77 .

\section{Acknowledgments}

We are extremely grateful to Laurent Kuras and Kevin Struhl for providing the triple HA1-tagged SPT15 plasmid, for sharing results prior to publication, and for invaluable assistance with the chromatin immunoprecipitation technique. We are also extremely grateful to Min-Hao Kuo and David Allis for providing the antiacetylated H3 antibodies and for helpful discussions. We thank Mary Bryk and Ting Wu for critical reading of the manuscript. This work was supported by a grant to F.W. from the National Institutes of Health (GM45720).

The publication costs of this article were defrayed in part by payment of page charges. This article must therefore be hereby marked "advertisement" in accordance with 18 USC section 1734 solely to indicate this fact.

\section{References}

Birck, C., O. Poch, C. Romier, M. Ruff, G. Mengus, A.C. Lavigne, I. Davidson, and D. Moras. 1998. Human TAF(II)28 and TAF(II) 18 interact through a histone fold encoded by atypical evolutionary conserved motifs also found in the SPT3 family. Cell 94: 239-249.

Blank, T.E., M.P. Woods, C.M. Lebo, P. Xin, and J.E. Hopper. 1997. Novel Gal3 proteins showing altered Gal80p binding cause constitutive transcription of Gal4p-activated genes in Saccharomyces cerevisiae. Mol. Cell. Biol. 17: 2566-2575.

Cosma, M.P., T. Tanaka, and K. Nasmyth. 1999. Ordered recruitment of transcription and chromatin remodeling factors to a cell cycle- and developmentally regulated promoter. Cell 97: 299-311.

Dedon, P.C., J.A. Soults, C.D. Allis, and M.A. Gorovsky. 1991. A simplified formaldehyde fixation and immunoprecipitation technique for studying protein-DNA interactions. Anal. Biochem. 197: 83-90.

Dudley, A.M., L.J. Gansheroff, and F. Winston. 1999. Specific components of the SAGA complex are required for Gen4and Gcr1-mediated activation of the his $4-912 \delta$ promoter in Saccharomyces cerevisiae. Genetics 151: 1365-1378.

Eisenmann, D.M., K.M. Arndt, S.L. Ricupero, J.W. Rooney, and F. Winston. 1992. SPT3 interacts with TFIID to allow normal transcription in Saccharomyces cerevisiae. Genes \& Dev. 6: 1319-1331.

Eisenmann, D.M., C. Chapon, S.M. Roberts, C. Dollard, and F. Winston. 1994. The Saccharomyces cerevisiae SPT8 gene encodes a very acidic protein that is functionally related to SPT3 and TATA-binding protein. Genetics 137: 647-657.

Giniger, E., S.M. Varnum, and M. Ptashne. 1985. Specific DNA binding of GAL4, a positive regulatory protein of yeast. Cell 40: $767-774$

Grant, P.A., L. Duggan, J. Côté, S.M. Roberts, J.E. Brownell, R. Candau, R. Ohba, T. Owen-Hughes, C.D. Allis, F. Winston, S.L. Berger, and J.L. Workman. 1997. Yeast Gen5 functions in two multisubunit complexes to acetylate nucleosomal histones: Characterization of an Ada complex and the SAGA
(Spt/Ada) complex. Genes \& Dev. 11: 1640-1650.

Grant, P.A., D.E. Sterner, L.J. Duggan, J.L. Workman, and S.L. Berger. 1998. The SAGA unfolds: Convergence of transcription regulators in chromatin-modifying complexes. Trends Cell Biol. 8: 193-197.

Hampsey, M. 1997. A SAGA of histone acetylation and gene expression. Trends Genet. 13: 427-429.

. 1998. Molecular genetics of the RNA polymerase II general transcriptional machinery. Microbiol. Mol. Biol. Rev. 62: 465-503.

Hampsey, M. and D. Reinberg. 1999. RNA polymerase II as a control panel for multiple coactivator complexes. Curr. Opin. Genet. Dev. 9: 132-139.

Horiuchi, J., N. Silverman, B. Pina, G.A. Marcus, and L. Guarente. 1997. ADA1, a novel component of the ADA/ GCN5 complex, has broader effects than GCN5, ADA2, or ADA3. Mol. Cell. Biol. 17: 3220-3228.

Ikeda, K., D.J. Steger, A. Eberharter, and J.L. Workman. 1999. Activation domain-specific and general transcription stimulation by native histone acetyltransferase complexes. Mol. Cell. Biol. 19: 855-863.

Johnston, M. and M. Carlson. 1992. Regulation of carbon and phosphate utilization. In The molecular and cellular biology of the yeast Saccharomyces: Gene expression, pp. 193-281. Cold Spring Harbor Laboratory Press, Cold Spring Harbor, NY

Koh, S.S., A.Z. Ansari, M. Ptashne, and R.A. Young. 1998. An activator target in the RNA polymerase II holoenzyme. Mol. Cell 1: 895-904.

Kuo, M.H., J. Zhou, P. Jambeck, M.E. Churchill, and C.D. Allis. 1998. Histone acetyltransferase activity of yeast Gen $5 p$ is required for the activation of target genes in vivo. Genes \& Dev. 12: 627-639.

Kuras, L. and K. Struhl. 1999. Binding of TBP to promoters in vivo is stimulated by activators and requires Pol II holoenzyme. Nature 399: 609-613.

Lavigne, A.C., Y.G. Gangloff, L. Carre, G. Mengus, C. Birck, O. Poch, C. Romier, D. Moras, and I. Davidson. 1999. Synergistic transcriptional activation by TATA-binding protein and hTAFII28 requires specific amino acids of the hTAFII28 histone fold. Mol. Cell. Biol. 19: 5050-5060.

Lee, T.I. and R.A. Young. 1998. Regulation of gene expression by TBP-associated proteins. Genes \& Dev. 12: 1398-1408.

Li, X.-Y., A. Virbasius, X. Zhu, and M. Green. 1999. Enhancement of TBP binding by activators and general transcription factors. Nature 399: 605-609.

Massari, M.E., P.A. Grant, M.G. Pray-Grant, S.L. Berger, J.L. Workman, and C. Murre. 1999. A conserved motif present in a class of helix-loop-helix proteins activates transcription by direct recruitment of the SAGA complex. Mol. Cell. 4: 6373.

Melcher, K. and S.A. Johnston. 1995. GAL4 interacts with TATA-binding protein and coactivators. Mol. Cell. Biol. 15: 2839-2848.

Natarajan, K., B.M. Jackson, E. Rhee, and A.G. Hinnebusch. 1998. yTAFII61 has a general role in RNA polymerase II transcription and is required by Gen $4 p$ to recruit the SAGA coactivator complex. Mol. Cell 2: 683-692.

Orlando, V. and R. Paro. 1993. Mapping polycomb-repressed domains in the bithorax complex using in vivo formaldehyde cross-linked chromatin. Cell 75: 1187-1198.

Ptashne, M. and A. Gann. 1997. Transcriptional activation by recruitment. Nature 386: 569-577.

Roberts, S.M. and F. Winston. 1997. Essential functional interactions of SAGA, a Saccharomyces cerevisiae complex of Spt, Ada, and Gen5 proteins, with the Snf/Swi and Srb/me- 
diator complexes. Genetics 147: 451-465.

Ryan, M.P., R. Jones, and R.H. Morse. 1998. Swi-Snf complex participation in transcriptional activation at a step subsequesnt to activator binding. Mol. Cell. Biol. 18: 1774-1782.

Selleck, S.B. and J. Majors. 1987a. Photofootprinting in vivo detects transcription-dependent changes in yeast TATA boxes. Nature 325: 173-177.

- 1987b. In vivo DNA-binding properties of a yeast transcription activator protein. Mol. Cell. Biol. 7: 3260-3267.

Som, T., K.A. Armstrong, F.C. Volkert, and J.R. Broach. 1988. Autoregulation of 2 micron circle gene expression provides a model for maintenance of stable plasmid copy levels. Cell 52: 27-37.

St. John, T.P. and R.W. Davis. 1981. The organization and transcription of the galactose gene cluster of Saccharomyces. J. Mol. Biol. 152: 285-315.

Sterner, D.E., P.A. Grant, S.M. Roberts, L.J. Duggan, R. Belotserkovskaya, L.A. Pacella, F. Winston, J.L. Workman, and S.L. Berger. 1999. Functional organization of the yeast SAGA complex: Distinct components involved in structural integrity, nucleosome acetylation, and TATA-binding protein interaction. Mol. Cell. Biol. 19: 86-98.

Strahl-Bolsinger, S., A. Hecht, K. Luo, and M. Grunstein. 1997. SIR2 and SIR4 interactions differ in core and extended telomeric heterochromatin in yeast. Genes \& Dev. 11: 83-93.

Swanson, M.S., E.A. Malone, and F. Winston. 1991. SPT5, an essential gene important for normal transcription in Saccharomyces cerevisiae, encodes an acidic nuclear protein with a carboxy-terminal repeat. Mol. Cell. Biol. 11: 3009-3019.

Wang, L., L. Liu, and S.L. Berger. 1998. Critical residues for histone acetylation by Gen5, functioning in Ada and SAGA complexes, are also required for transcriptional function in vivo. Genes \& Dev. 12: 640-653.

Winston, F., C. Dollard, and S.L. Ricupero-Hovasse. 1995. Construction of a set of convenient Saccharomyces cerevisiae strains that are isogenic to S288C. Yeast 11: 53-55.

Wu, Y., R.J. Reece, and M. Ptashne. 1996. Quantitation of putative activator-target affinities predicts transcriptional activating potentials. EMBO T. 15: 3951-3963.

Yano, K. and T. Fukasawa. 1997. Galactose-dependent reversible interaction of Gal3p with Gal80p in the induction pathway of Gal4p-activated genes of Saccharomyces cerevisiae. Proc. Natl. Acad. Sci. 94: 1721-1726.

Zaman, Z., A.Z. Ansari, L. Gaudreau, J. Nevado, and M. Ptashne. 1998. Gene transcription by recruitment. Cold Spring Harbor Symp. Quant. Biol. 63: 167-171. 


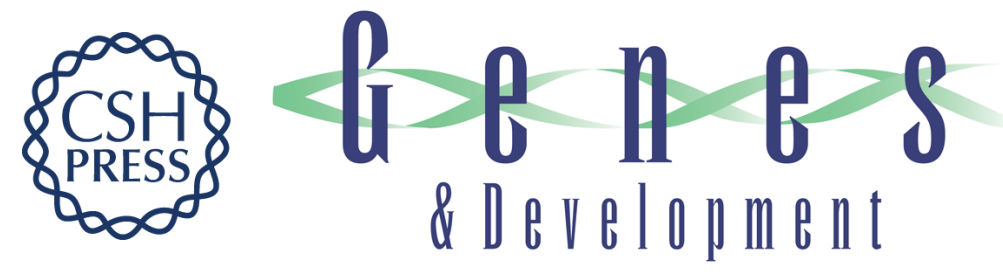

\section{The Spt components of SAGA facilitate TBP binding to a promoter at a post-activator-binding step in vivo}

Aimée M. Dudley, Claire Rougeulle and Fred Winston

Genes Dev. 1999, 13:

References This article cites 40 articles, 20 of which can be accessed free at:

http://genesdev.cshlp.org/content/13/22/2940.full.html\#ref-list-1

License

Email Alerting

Receive free email alerts when new articles cite this article - sign up in the box at the top

Service

right corner of the article or click here.

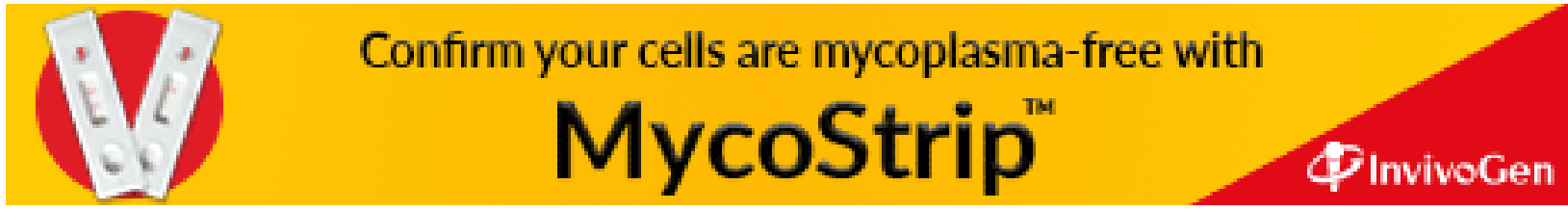

